\title{
Influencia de la industrialización en la salud ambiental: Visión histórica desde la revolución industrial a la pandemia por COVID-19
}

\section{Influence of industrialization on environmental health: Historical vision from the industrial revolution to the COVID-19 pandemic}

\author{
https://doi.org/10.52808/bmsa.7e5.61e2.001 \\ Roberto Carlos Dávila Morán ${ }^{1, *}$ \\ https://orcid.org/0000-0003-3181-8801 \\ Héctor Portillo Rios ${ }^{2}$ \\ https://orcid.org/0000-0003-1432-8472 \\ Leonardo Velarde Dávila ${ }^{3}$ \\ https://orcid.org/0000-0002-8096-0196 \\ Fernando Vásquez Perdomo ${ }^{4}$ \\ https://orcid.org/0000-0002-0537-447X \\ José Leonor Ruiz Nizama ${ }^{4}$ \\ https://orcid.org/0000-0003-0444-244X
}

Recibido: 07/03/2021

Aceptado: $21 / 06 / 2021$

\section{RESUMEN}

La contaminación ambiental, se inicia con la época industrial y se acrecienta luego de la segunda guerra mundial con el auge de la tecnología. En la década de los 70 el hombre comienza a tomar conciencia de la gravedad de este progresivo e irreparable daño. La ecología y ciencias afines se desarrollan y por doquier se implementa acciones para manejar o paliar el daño. La contaminación puede alterar directamente los componentes de un ecosistema, agua, aire o suelos, pero también puede darse por presencia de fenómenos físicos o físicoquímicos inusuales, como calor o radiación ionizante. Sus efectos se manifiestan principalmente por daño a los ecosistemas y por tanto daño a la salud de los seres humanos, animales, vegetales, la tierra o el agua (Nebel, 1999; Clark, 2003). Hoy son harto conocidos los desastres ecológicos de Londres, Donora, Minamata, Chernobyl, Bophal, del Golfo Pérsico, del Danubio originado en Baia Mare o los de petróleo en los mares (Baxter, 1991). En el Perú, sabemos del daño ambiental de la Ciudad de la Oroya, del lago de Junín, el derrame de Choropampa y la contaminación urbana por polvo de plomo a partir de los depósitos de concentrados de minerales en el Callao. De acuerdo con la Organización Panamericana de la Salud, hay 20. 000 casos confirmados de COVID-19 en la Cuenca del Amazonas (Martín, 2020). Mineros de oro y taladores ilegales están exponiendo a las comunidades indígenas a riesgos de salud considerables, una situación que podría tener consecuencias severas dadas la pobre capacidad de respuesta de los hospitales y servicios de salud en la región. Por tanto, la propagación del virus en estas comunidades podría implicar una tragedia que, además de las pérdidas humanas, podría, a su vez, afectar el conocimiento tradicional y producir efectos negativos en la gobernanza de la región. Como resultado, podría haber incluso mayor deforestación en el futuro. Políticas diferenciales para atender a las poblaciones étnicas en la región son una necesidad urgente (Bermudez et al., 2020; Vélez et al., 2020a). Es por ello que la presente investigación se planteo establecer la Influencia de la industrialización en la salud ambiental: Visión histórica desde la revolución industrial a la pandemia por COVID-19.

Palabras clave: Contaminación ambiental e influencia de la industrialización, Industrialización y salud ambiental, Revolución industrial y pandemia por Covid-19

\section{ABSTRACT}

Environmental pollution begins with the industrial era and increases after the Second World War with the rise of technology. In the 1970s, man began to become aware of the seriousness of this progressive and irreparable damage. Ecology and related sciences are developed and actions are implemented everywhere to manage or alleviate the damage. Pollution can directly alter the components of an ecosystem, water, air or soils, but it can also occur due to the presence of unusual physical or physicochemical phenomena, such as heat or ionizing radiation. Its effects are manifested mainly by damage to ecosystems and therefore damage to the health of human beings, animals, plants, land or water (Nebel, 1999; Clark, 2003). Today the ecological disasters of London, Donora, Minamata, Chernobyl, Bophal, the Persian Gulf, the Danube originating in Baia Mare or those of oil in the seas are well known (Baxter, 1991). In Peru, we know of the environmental damage of the City of Oroya, Lake Junin, the Choropampa spill, and urban contamination by lead dust from deposits of mineral concentrates in Callao. According to the Pan American Health Organization, there are 20,000 confirmed cases of COVID-19 in the Amazon Basin (Martín, 2020). Gold miners and illegal loggers are exposing indigenous communities to considerable health risks, a situation that could have severe consequences given the poor response capacity of hospitals and health services in the region. Therefore, the spread of the virus in these communities could imply a tragedy that, in addition to the human losses, could, in turn, affect traditional knowledge and produce negative effects on the governance of the region. As a result, there could be even more deforestation in the future. Differential policies to serve ethnic populations in the region are an urgent need (Bermudez et al., 2020; Vélez et al., 2020a). That is why this research set out to establish the Influence of industrialization on environmental health: Historical vision from the industrial revolution to the COVID19 pandemic.

Keywords: Environmental pollution and influence of industrialization, Industrialization and environmental health, Industrial revolution and pandemic by Covid-19 


\section{Introducción}

Es transcendental enfatizar, que la contaminación ambiental, se inicia con la época industrial y se acrecienta luego de la segunda guerra mundial con el auge de la tecnología. En la década de los 70 el hombre comienza a tomar conciencia de la gravedad de este progresivo e irreparable daño. La ecología y ciencias afines se desarrollan y por doquier se implementa acciones para manejar o paliar el daño. La contaminación puede alterar directamente los componentes de un ecosistema, agua, aire o suelos, pero también puede darse por presencia de fenómenos físicos o físicoquímicos inusuales, como calor o radiación ionizante. Sus efectos se manifiestan principalmente por daño a los ecosistemas y por tanto daño a la salud de los seres humanos, animales, vegetales, la tierra o el agua (Nebel, 1999; Clark, 2003). La enfermedad ambiental es producida por la exposición a agentes contaminantes presentes en el medio ambiente y que, a diferencia de lo que sucede con otras enfermedades, en ésta no existe primariamente predisposición genética del individuo. Generalmente, el término "enfermedad ambiental" se restringe a enfermedades no infecciosas causadas en su mayor parte por exposición a sustancias presentes en el ambiente, independiente del control inmediato que el enfermo haga sobre su entorno y a la restricción o eliminación de sus hábitos nocivos como fumar o uso de medicamentos, drogas o alcohol. Así, la enfermedad ambiental resulta en una categoría importante de patología referida a una colectividad como resultado de exposición a determinadoscontaminantes ubicuos en el medio (Ladou et al., 1999).

La Contaminación ambiental es definida como "todo cambio indeseable causado por el hombre en las características del aire, agua o suelo que afectan negativamente a los seres vivientes y en general al ecosistema”. El problema, como hemos visto, comienza con la revolución industrial a finales del siglo XVIII con el invento de la máquina a vapor y su hija inmediata, la máquina de tejer. Hasta entonces, la producción humana se hacía artesanalmente y la comercialización de los productos tenía cuasi un carácter de trueque. Se inicia así la gran etapa de la fábrica como sistema de producción masiva lo que trae avance tecnológico y desarrollo tan preciados hoy en día. Sin embargo, a resultas de lo mismo y concomitantemente otro proceso reservado y progresivo inicia y nos está llevando la decadencia de nuestro medio ambiente natural. En los años 40 del siglo pasado, se produce otra onda tecnológica, que algunos han considerado radical, la llaman "industria de guerra" y por supuesto en esos momentos graves a nadie le importa el deterioro del ambiente (Nebel, 1999; Jeyaratnam, 2000).

La atmósfera se deteriora por acumulación de gases llamados gases de efecto invernadero (GEI), resultantes del uso de combustibles fósiles, incremento desordenado de las industrias, expansión de la ganadería y deforestación galopante. De otro lado los cambios climáticos aumentan los episodios de contaminación atmosférica por incremento de los movimientos circulantes en la atmósfera y la consiguiente dispersión de los contaminantes. Los GEI más importantes son dióxido de carbono, oxido nitroso, metano y los fluoroclorocarbonos. Los GEI afectan la salud humana de dos maneras, primero indirectamente con el aumento de temperatura, humedad y lluvias en una determinada región, lo que favorece el desarrollo de enfermedades metaxénicas cuyos vectores dependen de la variación climática; segundo, directamente, pues conllevan aumento de enfermedades relacionadas de manera directa a la contaminación. Adicionalmente, la contaminación disminuye la capa de ozono e incrementa la radiación UV con aumento de lesiones dérmicas y oculares (Samel, 1997; Deulin, 19975).

En el recuento histórico, el concepto de enfermedad ambiental tuvo su origen en el reconocimiento de enfermedad ocupacional, quizá porque es más fácil correlacionar exposiciones intensas y sostenidas con determinada entidad nosológica, p. ej., neumoconiosis en exposición a polvo de sílice libre o cáncer de escroto en limpiadores de chimeneas expuestos a hollín. Muchas de estas enfermedades llamaron ya la atención en el inicio de la revolución industrial en el siglo XVIII. Hoy son harto conocidos los desastres ecológicos de Londres, Donora, Minamata, Chernobyl, Bophal, del Golfo Pérsico, del Danubio originado en Baia Mare o los de petróleo en los mares (Baxter, 1991). En el Perú, sabemos del daño ambiental de la Ciudad de la Oroya, del lago de Junín, el derrame de Choropampa y la contaminación urbana por polvo de plomo a partir de los depósitos de concentrados de minerales en el Callao. Además, en el interior, las ciudades pequeñas han comenzado a sentir el impacto del deterioro de su medio ambiente, pues de villas y villorrios pasan bruscamente a ciudades de industrialización emergente.

Desde hace algunas décadas, el aire de la mayoría de las capitales de los países latinoamericanos se ha deteriorado, como consecuencia de la instalación de industrias en su periferia y del rápido crecimiento del obsoleto parque automotor que usa combustible fósil con aditivos de plomo. La migración no planificada del campo a la ciudad contribuye alagravamiento por la tugurización de las ciudades de crecimiento intermedio. El fenómeno mundial de globalización y el desempleo son factores condicionantes que han llevado a la domiciliarización del trabajo. 
La diversidad de ecosistemas en el Perú y los grandes desafíos para conservarlos y gestionarlos adecuadamente hacen fundamental la investigación en temas ambientales (MINAN, 2013). Para enfocar mejor estos temas, se realizó del 28 al 30 de abril de 2014 en Lima un taller sobre el impacto en salud de la contaminación ambiental de agua, aire y cambio climático, donde los autores participaron. Para profundizar sobre el tema, se realizó una revisión narrativa de la literatura sobre el impacto en la salud que tiene la contaminación del aire y del agua de consumo humano, así como el efecto del Cambio climático en el Perú. En la región de las Américas, la OMS estima que el rotavirus es responsable de la mayoría de casos de enfermedad diarreica aguda (EDA) en niños $<5$ años, seguido por E. coli patógena (Lanata et al., 2013). La EDA también puede verse influenciada por factores de variabilidad climática en poblaciones pobres localizadas en zonas marginales (Bennett el al., 1997; Checkley et al., 2000;). Estudios recientes en Perú demuestran que las EDA afectan tanto el peso del niño como su crecimiento (Richard et al., 2013); igualmente, se ha demostrado en Perú que el acceso al agua y a la eliminación de excretas revierte el efecto adverso sobre el crecimiento de la estatura en niños quepreviamente tenían inadecuado acceso a agua segura o a eliminación de excretas 1997; Checkley et al., 2004; Checkley et al., 2003; Guerrant., 2013).

En la actualidad, la exposición a la contaminación del aire puede contribuir a la propagación de la enfermedad. Esto está basado en la posibilidad de que el virus SARS-CoV-2 esté presente en material particulado, como la evidencia preliminar lo sugiere (Setti et al., 2020). Si en realidad este fuera el caso, el virus podría viajar en la superficie de ese material particulado hasta los pulmones (Schraufnagel et al., 2019). A pesar de que el papel de la contaminación del aire en la transmisión por aire del virus todavía es incierto, resultados en China sugieren que existiría una relación positiva entre la presencia de contaminantes en el aire y la infección por COVID-19 (Zhu et al., 2020). Si periodos extensos de exposición a la contaminación incrementan el riesgo asociado de contraer la enfermedad (Wu et al., 2020; Zhu et al., 2020), los trabajadores informales podrían ser una de las poblaciones más vulnerables.

Para Bolivia, Brasil, Colombia, Ecuador y Perú, la región Amazónica también es muy importante en términos de diversidad étnica. Lo mismo es cierto para algunos bosques localizados en Costa Rica, Guatemala, Honduras, México, Nicaragua y Panamá. Estudios recientes han documentado el impacto de las reservas indígenas y étnicas en la reducción tanto de la deforestación como de la emisión de carbono en el Amazonas (Blackman et al., 2017; Blackman y Veit, 2018; Herrera et al., 2019) y en otros bosques de la región (Vélez et al., 2020b). La propagación del virus y el impacto de la pandemia en las comunidades étnicas de estas y otras regiones es una fuente importante de preocupación (Bermudez et al., 2020). En Ecuador, la Región Amazónica registra 10. 265 casos de COVID-19 acumulados desde el inicio de la pandemia, esa cifra hace que esta región tenga la mayor tasa de incidencia del virus en el país (Ministerio de Salud Pública, 2020).

De acuerdo con la Organización Panamericana de la Salud, hay 20. 000 casos confirmados de COVID-19 en la Cuenca del Amazonas (Martín, 2020). Mineros de oro y taladores ilegales están exponiendo a las comunidades indígenas a riesgos de salud considerables, una situación que podría tener consecuencias severas dadas la pobre capacidad de respuesta de los hospitales y servicios de salud en la región. En Colombia, la mayoría de los departamentos de la región del Amazonas carecen de unidades de terapia intensiva (El Tiempo, 28 de marzo de 2020; Cárdenas y Montoya, 2020; Bermudez et al., 2020). Por tanto, la propagación del virus en estas comunidades podría implicar una tragedia que, además de las pérdidas humanas, podría, a su vez, afectar el conocimiento tradicional y producir efectos negativos en la gobernanza de la región. Como resultado, podría haber incluso mayor deforestación en el futuro. Políticas diferenciales para atender a las poblaciones étnicas en la región son una necesidad urgente (Bermudez et al., 2020; Vélez et al., 2020a). Es por ello que la presente investigación se planteo establecer la Influencia de la industrialización en la salud ambiental: Visión histórica desde la revolución industrial a la pandemia por COVID19.

\section{Visiones históricas y contemporáneas de la industrialización y la salud ambiental}

\section{Antes de 1945: Riesgo sanitario microbiológico}

Nos permitimos poner de relieve, que la Salud Ambiental inició con dos grandes revoluciones: la agrícola y la industrial en 1712. Un siglo después, y a partir del deterioro acelerado del ambiente, inició el abordaje de la salud de las poblaciones (Garibay y Curiel, 2005). Por tanto, el cambio en los espacios laborales que va del agrícola al industrial desencadenó una serie de riesgos sanitarios nuevos. Los orígenes de la salud ambiental podrían ser muy antiguos y remontarse a las primeras interacciones y desafíos entre el ser humano y su entorno natural en la búsqueda permanente del bienestar, un punto clave de surgimiento del campo se remite a los "despertares industriales" (Frumkin, 2005) y los movimientos poblacionales de los pueblos a las ciudades. Podemos decir que desde el siglo XVIII, el hacinamiento tanto en las moradas habitacionales como en las industrias provocó un grave deterioro en el ambiente, y como consecuencia, daños a la salud humana. Los problemas sanitarios (casas y lugares de trabajo sin ventilación y húmedas, calles contaminadas con excremento, agua estancada y suministros de agua inadecuados) se acrecentaron exponencialmente. Todo parecía indicar que el avance tecnológico y de producción era inversamente proporcional al 
estado de deterioro de la salud humana y del ambiente. Las condiciones ambientales insalubres existieron a la par de las inadecuadas condiciones sociales. Asï como también, las jornadas laborales eran muy largas y los salarios muy bajos. Como resultado, los trabajadores sufrían de malnutrición y mala condición física, la cual se vio agravada por las fábricas mal ventiladas, las altas temperaturas y la humedad, ocasionando predisposición a contraer infecciones de tipo bacterianas (Foskket, 1999). Inglaterra, país que inició con el proceso de industrialización y en consecuencia el que conoció los primeros efectos adversos a la salud, pero también fue el pionero en emprender la búsqueda de estrategias de solución. Mientras en Inglaterra la salud ocupacional había constituido todo un tema de abordaje desde las primeras décadas del siglo XIX, en Estados Unidos no es sino hasta el siglo XX cuando, gracias a la iniciativa de Alice Hamilton (1969-1970), se empiezaron a documentar los vínculos entre exposiciones tóxicas y las enfermedades de trabajadores como mineros, comerciantes y obreros industriales (Frumkin, 2005). Durante esta etapa predominó un concepto de salud como ausencia de enfermedad, y la idea que la enfermedad es el resultado de la contaminación microbiologíca. Asimismo, la percepción de ambiente se remitió a "todo lo que rodea" al ser humano sin ser parte del mismo, y se planteó un enfoque utilitario de la naturaleza como proveedora de los recursos naturales.

\section{De 1945 a 1956: Riesgo sanitario y quimico toxicológico: de 1945 a 1956}

El pasado siglo XX aportó numerosos ejemplos de la enérgica implementación de procesos de industrialización en diversos países y territorios en aras de lograr un rápido desarrollo industrial y económico, pero sin considerar en su justa magnitud sus posibles impactos en el ambiente, cuyas consecuencias sobre la salud resultaron a veces rápidamente evidentes. Algunas de ellas son la denominada "asma epidémica en la ciudad de Yokkaichi", posterior a la construcción de un gran complejo petroquímico; la intoxicación crónica por metil mercurio en pescado (enfermedad de Minamata) o la intoxicación crónica por cadmio, caracterizada por daño renal, fragilidad ósea y dolor en el agua de regadío del arroz, denominada "itai - itai” (dolor, dolor), episodios todos ocurridos en Japón, en la década siguiente a la Segunda Guerra Mundial. Estos ocasionaron severos impactos en la salud pública, así como elevados costos económicos por la implementación de las medidas para la mitigación de sus consecuencias, muy superiores a los beneficioseconómicos iniciales (Yassi et al., 2008). La exposición a sustancias y preparados químicos peligrosos, el uso de biocidas y plaguicidas fitosanitarios, las sustancias CMR (carcinógenas, mutágenas y tóxicas para la reproducción), los COV (compuestos orgánicos volátiles)20, las sustancias PBT (persistentes bioacumulables y tóxicas), las dioxinas 21 y furanos, los PCB, los retardantes de llama, los alteradores endocrinos 22 representan amenazas que deben ser objeto de medidas de evaluación, y de reducción y control del riesgo tal y como se establecen en sus respectivas legislaciones (Kogevinas y Jane, 2000; Olea et al., 2002; Porta et al., 2002).

Asimismo, la presencia en la atmósfera de sustancias o energía en tales cantidades y de tal duración que son capaces de causar daños a los hombres, a las plantas o a la vida animal, o de dañar los objetos y estructuras fabricadas por el hombre, o de provocar cambios de temperatura y clima, o dificultades para el cómodo disfrute de la vida, de los bienes o de otras actividades humanas (Elsom, 1990). Es importante destacar, que existen centenares de sustancias que producen la contaminación del aire: el $80 \%$ de estos son invisibles ante nuestros ojos y la mayoría son inodoros, por lo tanto el riesgo de exponerse a sus efectos es grande. Hasta los años sesenta, los contaminantes sólo eran contemplados como un problema en proximidad con fuentes de emisión individuales dentro o cerca de áreas urbanas. Posteriormente, los estudios demostraron que los contaminantes habían sido transportados a largas distancias y habían causado diversos efectos en el entorno de lugares muy distantes de la fuente de emisión. Los cinco contaminantes atmosféricos más comunes en el medio urbano son: partículas, óxidos de azufre, monóxido de carbono, óxidos de nitrógeno, e hidrocarburos (Dufus, 1983; Elsom, 1990).

La contaminación ambiental urbana originada en las actividades humanas está lejos de representar un fenómenoreciente ya que éste se suscitó con la industrialización de la sociedad en el siglo dieciocho. Sin embargo, han sidolos episodios sin precedentes de alta contaminación ambiental durante los años 1940 y 1950 en ciudadesnorteamericanas y europeas los que han permanecido como símbolos históricos de un pasado poluido, acompañado de muertes y enfermedades. En el siglo veinte, se distinguen en especial las letales neblinasinvernales londinenses las que después de la Segunda Guerra Mundial pasaron a ser densas, olorosas y altamente desagradables. Estos han sido eventos extremos de contaminación que fueron causados por el alto contenido de dióxido de sulfuro y de partículas en el aire, provenientes éstos del uso decarbón como combustible tanto en fábricas como en viviendas, junto a las bajas temperaturas reinantes.

Como consecuencia de condiciones medio ambientales tan deplorables, se introdujo, a la par de nuevas tecnologías máseficientes y nuevos materiales menos polucionantes en la producción industrial y en el uso de la energía, y eltraslado de muchas de las plantas manufactureras fuera de los confines urbanos, el establecimiento deregulaciones nacionales para el cuidado ambiental desde los años 1970, como las actas sancionadas en EE. UU yen el Reino Unido. Todos estos cambios produjeron un drástico mejoramiento de la calidad del aire urbano (Brimblecombe, 1988). A pesar de los notorios cambios en la política ambiental, los avances tecnológicos y la reorganización industrial, 
la polución ambiental urbana continúa, representando una problemática no solucionada y peligrosa para el bienestar común (Chemi, 2001).

\section{De 1957 a 1984: Riesgo socio organizativo, rumbo a la urbanización acelerada}

El crecimiento de la población ha sido una de las fuerzas más frecuentemente citadas para explicar la sobreexplotación de los recursos naturales y la degradación ambiental (McNeill, 2006). El explosivo crecimiento poblacional mundial observado durante el siglo XX, ha sido determinante de la condición global actual. No obstante, también se reconoce que el crecimiento per se no es el único factor que determina la fuerza de la presión que se ejerce sobre el ambiente y los recursos naturales. La capacidad económica de consumo de la sociedad tiene también una importante influencia, así como la eficiencia técnica con la que se usan los recursos para la producción de los satisfactores (Ehrlich et al., 1971; York et al., 2003). Otras variables como la desigualdad, el nivel de urbanización, el régimen jurídico y la institucionalidad también modifican la dinámica de las causas subyacentes de la presión ambiental (De Sherbinin et al., 2007).

Otros problemas más complejos pueden tener lugar a consecuencia del rápido y desmesurado crecimiento demográfico en urbanizaciones carentes de un plan director que garantice los recursos e infraestructuras necesarias para la satisfacción de las necesidades básicas de las nuevas poblaciones y distritos urbanos, tales como las necesidades de viviendas, fuentes de abasto, sistemas de tratamiento de agua, la disposición de residuales y demás elementos que garanticen las acciones básicas de saneamiento ambiental, las redes viales que den respuesta al incremento del tráfico, entre otros, así como nuevas transformaciones en el uso del suelo, la demanda de nuevas infraestructuras y equipamientos de los servicios de alimentación, educación y salud, por citar algunos de los principales (Yassi et al., 2008).

El caso de la contaminación del aire urbano en las últimas décadas y el impacto que éste ha tenido en la salud humana es precisamente un ejemplo de la persistencia de la degradación ecológica y su relación con un tipo de sociedad en particular. Mientras que las evidencias científicas sólo nos indican cuán serio es el problema de la contaminación local del aire, es, se demuestra que este fenómeno es, además, muy preocupante, pues se ha globalizado, a la vez que estos impactos físicos dependen en gran medida de las formas políticas y económicas de la sociedad. El impacto ambiental negativo asociado con el crecimiento económico y sus consecuencias humanas son parecidos, si no iguales, en muchos de los lugares donde éste ocurre, el crecimiento se ha caracterizado, por ejemplo, por la producción, comercialización y uso de enormes cantidades de energía de origen fósil, que a través de procesos químicos han impactado directamente a la ecología. A su vez, ha avanzado la internacionalización del proceso de contaminación del aire y de la asociada insalubridad, predisponiendo a la población a enfermedades respiratorias, como el asma y la bronquitis (Chemi, 2001)

\section{De 1985 a 2019: Riesgo de cambios globales}

Los temas relacionados con el comercio internacional, la inversión y las actividades económicas sectoriales, tale como la demanda de energía, transporte y agricultura son áreas muy visibles del proceso de globalización y suimpacto ambiental (OECD, 1998). Las instalaciones centrales de producción energética en gran escala -tantocomo de poder nuclear, inmensas represas hidroeléctricas, y plantas generadoras de electricidad- son necesariasen una economía global que pregona el crecimiento, el alto consumo y desperdicio y el transporte a largadistancia prácticamente de cada producto destinado al consumidor. Este crecimiento obliga a los paísesdesarrollados a expandir su gigantesca infraestructura y aumentar la producción de energía; pero la mayoría de las nuevas instalaciones se construyen en los países del Sur, pretendiendo de esta forma integrar a tales paísesen la economía global (Gorelick, 1999). La contaminación originada por enormes plantas ha contribuido a aumentar significativamente la concentración global de gases como el CO2 y ozono. Por ejemplo, cuando laconcentración pre-industrial de CO2, en partes por billón, era de 280.000 mundialmente, la presenteconcentración es de 363.000 ppb, el presente nivel de crecimiento es de $0.5 \%$ anual, y su relativa contribución alefecto invernadero es del $60 \%$.

En el centro de las relaciones entre la globalización y el medio ambiente se encuentra la competitividadinternacional, que se fomenta para atraer el mayor número posible de inversiones extranjeras a un país. Lacompetitividad se manifiesta en dos maneras bastante opuestas. La primera es que las tendencias de expansiónde la globalización económica generan presiones a través de las empresas transnacionales para disminuir elpoder de las políticas nacionales que pudiesen proteger al medio ambiente en tanto que esta protección causaseuna disminución directa o indirecta de la rentabilidad para firmas locales o internacionales. La segunda forma esque la competitividad internacional en el marco de la globalización puede llegar a representar una oportunidadpara obtener algún grado de protección para el medio ambiente en la medida que políticas ambientalistasnacionales o regionales sean empleadas como una manera de mejorar la competitividad nacional y para atraernuevas inversiones de capital. Como parte de la liberalización de los mercados de inversión, y los esfuerzos para mejorar la calidad de vida paraaumentar la competitividad entre las regiones en el Norte., estos países han puesto en vigencia disposicionesambientales locales más 
severas pero que a la vez permiten la exportación de las fuentes contaminantes. Parte de las denominadas industrias sucias, ha sido transferida al Tercer Mundo (Gouldson and Murphy, 1997). Se establecio, la reubicación de las industrias fabricantes de amianto desde Estados Unidos hacia Latino américa, siendo Brasil y Méjico los receptores más frecuentes. Ya en los años 1980, un estudio mundial de 54 ciudades demostró que a pesar de que los niveles promedios de contaminación sulfúrica se encontraban dentro de los límites establecidos por la Organización Mundial (OMS) de la Salud, los niveles máximos de contaminación recomendados por ésta, eran excedidos en zonas urbanas dentro de numerosas ciudades tal que 625 millones de habitantes vivían en zonas donde la concentración de SO2 sobrepasaba los patrones de la OMS (WHO \& UNEP, 1987).

El crecimiento de la población ha sido una de las fuerzas más frecuentemente citadas para explicar la sobreexplotación de los recursos naturales y la degradación ambiental (McNeill, 2006). El explosivo crecimiento poblacional mundial observado durante el siglo XX, ha sido determinante de la condición global actual (ver más adelante el recuadro Tendencias de la población mundial). No obstante, también se reconoce que el crecimiento per se no es el único factor que determina la fuerza de la presión que se ejerce sobre el ambiente y los recursos naturales. La capacidad económica de consumo de la sociedad tiene también una importante influencia, así como la eficiencia técnica con la que se usan los recursos para la producción de los satisfactores (Ehrlich et al., 1971; York et al., 2003). Otras variables como la desigualdad, el nivel de urbanización, el régimen jurídico y la institucionalidad también modifican la dinámica de las causas subyacentes de la presión ambiental (De Sherbinin et al., 2007).

La acción global es inaplazable, si también se toma en cuenta que la evidencia científica sugiere que, por un lado, ya se han rebasado los "límites planetarios" de procesos tan importantes como los ciclos del nitrógeno y del fósforo y de erosión de la diversidad genética; y por otro, que se han incrementado los riesgos de sobrepasarlos en el caso del cambio climático, el cambio de uso del suelo y la biodiversidad (Steffen et al., 2015). Si bien es cierto, que no se tiene certeza de la intensidad y naturaleza de los efectos que esto podría ocasionar, es muy probable violar los límites del sistema climático y de la integridad de la biosfera, considerados como los dos núcleos del sistema planetario, que podrían trasladarnos a un nuevo orden en el planeta (Steffen et al., 2015), con graves consecuencias económicas y sociales que pueden extenderse no solamente a las condiciones actuales y de mediano plazo, sino involucrar el bienestar de las siguientes generaciones. El impacto ecológico contemporáneo ha sido evidente en partes muy diferentes y también alejados entre sí, pero en especial allí donde se ha promovido el rápido crecimiento económico y la urbanización; donde ha predominado el crecimiento del sector terciario; también donde la industria pesada se encuentra, o se encontraba, en plena actividad; y donde la actividad económica e industrial han disminuido drásticamente, dejando atrás, sí un legajo dedesechos tóxicos. En la actualidad, el proceso de globalización se refleja en prácticas locales y urbanas asociadas a la reestructuración y expansión de actividades económicas, en su mayoría sin consideración del efecto ecológico que éstas causan.

En el Perú, un tercio de las residencias utilizan combustible de biomasa para cocinar o para calefacción (INEI, 2007). Los contaminantes producidos por la combustión de esta biomasa tienen efectos negativos para la salud (Yucra et al., 2011; Sukhsohale et al., 2013). El aire exterior está contaminado, a un alto nivel comparado con otros países latinoamericanos, según reportes de la Organización Mundial de la Salud (OMS), se conoce, que la materia particulada en el aire exterior está asociada con varias enfermedades crónicas. El Perú es también un país netamente minero, por lo cual está expuesto a la contaminación ambiental producida por la explotación minera (Astete, 2009); esto implica un importante efecto negativo tanto de la contaminación de aire de exteriores y de interiores. La diversidad de ecosistemas en el Perú y los grandes desafíos para conservarlos y gestionarlos adecuadamente hacen fundamental la investigación en temas ambientales (MINAN, 2013). Para enfocar mejor estos temas, se realizó del 28 al 30 de abril de 2014 en Lima un taller sobre el impacto en salud de la contaminación ambiental de agua, aire y cambio climático, donde los autores participaron. Para profundizar sobre el tema, se realizó una revisión narrativa de la literatura sobre el impacto en la salud que tiene la contaminación del aire y del agua de consumo humano, así como el efecto del cambio climático en el Perú.

\section{Desde diciembre 2019: la crisis sanitaria mundial "Pandemia por COVID-19"}

Los impactos negativos que la contaminación del aire tiene en la salud han sido documentados de manera extensa. Este entra en el cuerpo a través del tracto respiratorio, ocasionando efectos sistémicos que pueden causar enfermedades en otros órganos (Schraufnagel et al., 2019). La contaminación del aire está ligada a muchos problemas de salud, incluso causando la muerte prematura en niños y adultos (Chay y Greenstone, 2003; Curtis et al., 2006). Sin embargo, la exposición al aire contaminado y, por ende, sus efectos negativos, no está uniformemente distribuida entre la población. La exposición continua a la contaminación del aire es particularmente problemática para una región en la que una parte significativa de la población económicamente activa pertenece al sector informal. Los más acaudalados pueden mudarse lejos de las áreas altamente contaminadas, ya sea temporal o permanentemente (Shen et al., 2020). Algunos incluso están en condiciones de cambiar de trabajo o comprar equipos de alta calidad para su protección (máscaras de aire o purificadores de ambiente). En contraste con esta situación, las personas de bajos ingresos, viven en 
áreas de mayor densidad de tráfico o cerca de fuentes de contaminación (plantas de energía o complejos industriales), tienen que caminar distancias largas para tomar el transporte público o incluso trabajan en las calles (Neidell, 2004; Calderón et al., 2012; Schraufnagel et al., 2019). Lo mismo suele ser cierto con relación a su exposición a la contaminación de aire en interiores, debido a que el uso de leña está negativamente correlacionado con el ingreso; la exposición de largo plazo a la contaminación en interiores puede ser particularmente alta para las mujeres, quienes están más involucradas en el trabajo doméstico (Jeuland et al., 2015).

Es importante destacar, que contaminación del aire puede ser una causa significativa de las condiciones preexistentes asociadas a una mayor mortalidad por COVID-19, se ha argumentado que la exposición prolongada a este tipo de contaminación, ha hecho a la población más vulnerable a la enfermedad (Wu et al., 2020). En el caso de la Zona Metropolitana del Valle de México, se ha encontrado que, efectivamente, existe una relación positiva entre la exposición prolongada a $\mathrm{PM}_{2.5}$ y la probabilidad de morir por COVID-19 (López et al., 2020). La exposición actual a la contaminación del aire puede contribuir a la propagación de la enfermedad. Esto está basado en la posibilidad de que el virus SARS-CoV-2 esté presente en material particulado, como la evidencia preliminar lo sugiere (Setti et al., 2020). Lo que indica, que el virus podría viajar en la superficie de ese material particulado hasta los pulmones (Schraufnagel et al., 2019). A pesar de que el papel de la contaminación del aire, la transmisión por aerea del virus todavía es incierto, resultados en China sugieren que existiría una relación positiva entre la presencia de contaminantes en el aire y la infección por COVID-19 (Zhu et al., 2020), sugiriendo que periodos extensos de exposición a la contaminación incrementan el riesgo asociado de contraer la enfermedad (Wu et al., 2020; Zhu et al., 2020), los trabajadores informales podrían ser una de las poblaciones más vulnerables.

Las restricciones para controlar y reducir el movimiento entre y a través de las áreas urbanas de la región han disminuido la actividad económica y el uso de automóviles, camiones y otros vehículos motorizados. Como resultado, al igual que en otras grandes urbes del mundo, muchas ciudades de América Latina han experimentado una reducción de la contaminación del aire en el corto plazo. Las concentraciones de $\mathrm{NO}_{2}$, se han reducido considerablemente en ciudades por toda la región, comparadas con los niveles observados durante los primeros 10 días de marzo (previo a las medidas de confinamiento). Bogotá muestra la mayor reducción (-83\%), mientras que en Guayaquil, Río de Janeiro y Sao Paulo fue menor al 30\%. En Bogotá, las emisiones de $\mathrm{PM}_{2.5}$ disminuyeron en $60 \%$ (Secretaría del Medio Ambiente del Distrito de Bogotá, comunicación personal, 3 de junio de 2020), mientras que los niveles de $\mathrm{PM}_{10}, \mathrm{PM}_{2.5}$ y CO se redujeron en al menos 50\%. Observandose el mismo resultado en la ciudad de Buenos Aires comparados con el mismo periodo del año anterior (Rocha, 2020). Mientras tanto, en Quito, la ciudad más poblada de Ecuador, las concentraciones de $\mathrm{PM}_{10}$ y $\mathrm{PM}_{2.5}$ tuvieron una caída sustancial promedio al día de $25-50 \mu \mathrm{g} / \mathrm{m} 3$ dos meses antes de la pandemia a 4-7 $\mu \mathrm{g} / \mathrm{m} 3$ durante la cuarentena (Roa, 2020). Sin embargo, en algunas ciudades de América Latina los cambios observados en las concentraciones de algunos contaminantes son relativamente pequeñas. Estas concentraciones son el resultado de un complejo proceso que, además de los niveles de emisión, dependen, entre otras cosas, de las condiciones atmosféricas, las características topográficas y la interacción entre diferentes compuestos y contaminantes. En Ciudad de México, a pesar de que la congestión por tráfico ha decrecido (IADB y IDB Invest, 2020), las reducciones en $\mathrm{SO}_{2}, \mathrm{PM}_{2.5}$ y $\mathrm{PM}_{10}$ han sido modestas, y no ha habido una reducción en el ozono. Mientras tanto, en Río de Janeiro, el confinamiento parcial de la población llevó a un incremento en las concentraciones de ozono (Dantas et al., 2020).

Otros eventos, como, por ejemplo, incendios forestales y condiciones climáticas, podrían también tener efectos importantes en la calidad del aire. Bogotá y otras ciudades en Colombia experimentaron varios eventos de empeoramiento de la calidad del aire durante el confinamiento, aparentemente debido a incendios forestales de Venezuela y otras regiones del país. A pesar de que la movilidad en algunas de las principales ciudades de la zona central y sur de Chile ha disminuido bajo el confinamiento, la calidad del aire podría empeorar durante los meses de invierno. Muchas familias en esta región usan leña como calefacción y, conforme pasen más tiempo en el interior, podría haber un incremento en la concentración de material particulado tanto en el interior como en el exterior (Encinas et al., 2020). Se espera observar un efecto similar conforme se profundice la crisis y la pandemia alcance las zonas rurales. En México, aproximadamente 28 millones de personas viven en hogares que utilizan leña como su medio de energía principal o secundario para cocinar (Inegi, 2019). Como tal, están crónicamente expuestos a la contaminación interior y son particularmente vulnerables al COVID-19.

Como consecuencia de la pandemia, el ingreso de muchos hogares, ubicados en áreas rurales y periurbanas, se ha visto afectado de forma negativa. La reducción en el ingreso, así como problemas con la distribución de gas, en comunidades relativamente aisladas, ha llevado a un incremento en el uso de leña (Masera et al., 2020), lo que a su vez podría incrementar el riesgo asociado al COVID-19. En cualquier caso, es poco probable que las mejoras en la calidad del aire observadas en algunas ciudades de América Latina sean sostenibles en el tiempo. Los niveles de contaminación previos al confinamiento podrían regresar de manera paulatina conforme se vayan relajando las medidas de confinamiento y las ciudades recuperen su movilidad y retomen actividades económicas. Todavía está por verse si algunas de las medidas de movilidad implementadas en ciudades como Bogotá, Quito y Ciudad de México 
permanecerán en el largo plazo (vías exclusivas para bicicletas y zonas para peatones más amplias). Lo mismo es cierto para el incremento en el uso de bicicletas observado en Costa Rica como resultado de las restricciones del gobierno al uso de automóviles privados durante ciertas horas del día. Más allá de las mejoras en la calidad del aire, es posible que algunos trabajadores, después de haberse visto obligados a utilizar bicicletas, mantengan este hábito como alternativa de transporte en el largo plazo. Un factor importante que podría incrementar y preservar este efecto es la implementación de políticas públicas que incentiven e incluso subsidien el uso de bicicletas como medio de transporte. Sin embargo, antes de promover dichas políticas, es recomendable que se analicen los efectos que una mayor exposición a la contaminación ambiental puede tener en la salud de los que usan la bicicleta como medio de transporte.

Los bosques son esenciales para la preservación de la biodiversidad y la regulación del clima. En particular, el Amazonas es crucial para mantener la salud del planeta debido a su rol fundamental en la regulación del clima de la Tierra (Ellwanger et al., 2020). Además de ser una fuente de alimento, medicinas y materia prima, los bosques juegan un papel clave en la subsistencia de los hogares rurales (Angelsen y Wunder 2003). La extracción de productos no maderables (animales salvajes, plantas y hongos silvestres) puede ser una fuente importante de ingreso y proveer parte de la dieta diaria para muchos hogares en situación de vulnerabilidad (Angelsen y Wunder 2003), de manera que mitigue la pobreza y reduzca la desigualdad (López y Taylor, 2007). Los Ecosistemas boscosos bien conservados reducen la prevalencia de enfermedades (Dunn 2010; Bauch et al., 2015). En contraste, la deforestación y los cambios del uso del suelo pueden alterar las interacciones entre el hombre y los animales, generando el potencial para la transmisión de infecciones zoonóticas de las poblaciones animales a las humanas, tal como parece haber ocurrido con el COVID-19; aproximadamente el 75\% de las infecciones emergentes son zoonóticas (Myers et al., 2013). Aún es pronto para realizar una evaluación formal de los efectos de la pandemia en la deforestación y el cambio en el uso de suelo en la región. Sin embargo, la información disponible sugiere que es probable que las medidas adoptadas para manejar el COVID-19 tengan efectos negativos en la cobertura forestal. En México, informes de diferentes regiones del país (Hidalgo, Tlaxcala, Chihuahua y la Península de Yucatán) dan cuenta de un incremento en la deforestación ilegal en los últimos dos meses. Señales tempranas de deforestación en Perú muestran cómo a pesar de que la deforestación en el periodo se ha reducido entre el 15 de marzo y el 15 de abril, después de esta fecha se incrementó sobrepasando los niveles observados en el mismo periodo en el 2018. En general, las tasas de deforestación en los países del Amazonas estaban en aumento antes de la emergencia por el COVID-19, una tendencia que la pandemia y el confinamiento asociado no ayudarán a revertir. Por el contrario, la reducción en los esfuerzos de monitoreo a lo largo de la región durante la pandemia podría estar asociada con un incremento en la tala de bosques y las emisiones de carbono debido a los cambios en el uso del suelo.

De acuerdo con datos del Instituto Nacional de Investigación Espacial de Brasil (INPE), el primer cuarto del 2020 ya evidenciaba un alza del $50 \%$ en el número de hectáreas deforestadas comparado con las estadísticas del año anterior (CNN, 2020). Las cifras de abril 2020 refuerzan esta tendencia con un incremento del 64\% con respecto a abril del 2019. De enero a abril del 2020, las alertas por deforestación en territorios indígenas aumentaron 59\% al compararlas con el mismo periodo del año anterior. A pesar de que en este momento no se pueden atribuir estos incrementos a las medidas tomadas por la pandemia, con seguridad esta no parece haber generado incentivos para detenerlos. Adicionalmente, otra preocupación para Brasil y otros países de la región, como, por ejemplo, Colombia, es que un aumento en deforestación puede derivar en aún más incendios forestales que los acontecidos el año anterior. De hecho, la evidencia muestra que en Colombia el confinamiento que ha acompañado a la pandemia está asociado con un incremento en incendios forestales (Amador et al., 2020). Contrario a otros países de la zona del Amazonas, Colombia mostró una reducción en deforestación en el 2019 comparado con el 2018 (Ideam, 2020). Sin embargo, el 2020 inició con una tendencia al alza y el confinamiento parece haber agravado la situación. La ausencia de monitoreo ambiental durante la pandemia parece haber incentivado las actividades de grupos armados y mafias regionales, exacerbando la deforestación e intensificando actividades ilegales de las que estos actores derivan ingreso, tales como la minería ilegal, el acaparamiento de tierras y los cultivos ilícitos (BBC Future, 2020). Para Bolivia, Brasil, Colombia, Ecuador y Perú, la región Amazónica también es muy importante en términos de diversidad étnica. Lo mismo es cierto para algunos bosques localizados en Costa Rica, Guatemala, Honduras, México, Nicaragua y Panamá. Estudios recientes han documentado el impacto de las reservas indígenas y étnicas en la reducción tanto de la deforestación como de la emisión de carbono en el Amazonas (Blackman, et al., 2017; Blackman y Veit, 2018; Herrera et al., 2019) y en otros bosques de la región (Vélez, et al., 2020b). La propagación del virus y el impacto de la pandemia en las comunidades étnicas de estas y otras regiones es una fuente importante de preocupación (Bermudez et al., 2020). En Ecuador, la Región Amazónica registra 10265 casos de COVID-19 acumulados desde el inicio de la pandemia, esa cifra hace que esta región tenga la mayor tasa de incidencia del virus en el país (Ministerio de Salud Pública, 2020).

\section{Conflictos de intereses}

Ninguno para declarar 
Agradecimiento

A Dios y nuestras instituciones de educación universitarias, nuestras casa de los saberes.

\section{Referencias}

Angelsen, A., \& Wunder, S. (2003). Exploring the forest-poverty link: Key concepts, issues and research implications (Occasional Paper, 40). Center for International Forestry Research. Bogor, Indonesia. Dispomible en: https://www.cifor.org/publications/pdf_files/occpapers/op-40.pdf (Acceso marzo 2021)

Astete, J., Cáceres, W., Gastañaga, M. del C., Lucero, M., Sabastizagal, I., Oblitas, T., Pari, J., \& Rodríguez, F. (2009). Intoxicacion por plomo y otros problemas de salud en ninos de poblaciones aledañas a relaves mineros. Rev Peru Med Exp Salud Pública, 26(1):15-9.

Bauch, S. C., Birkenbach, A. M., Pattanayak, S. K., \& Sills, E. O. (2015). Public health impacts of ecosystem change in the Brazilian Amazon. Proceedings of the National Academy of Sciences of the United States of America, 112(24):7414-7419. https://doi.org/10.1073/pnas.1406495111

Baxter P. J. (1991). Major chemical disasters. BMJ (Clinical research ed.), 302(6768):61-62. https://doi.org/10.1136/bmj.302.6768.61

BBC News. (2020). Coronavirus en Ecuador: las multitudinarias protestas por las drásticas medidas económicas y recortes de Lenín Moreno. Dispomible en: https://www.bbc.com/mundo/noticias-america-latina-52814371 (Acceso marzo 2021)

Bennett, A., Epstein, L. D., Gilman, R. H., Cama, V., Bern, C., Cabrera, L., Lescano, A. G., Patz, J., Carcamo, C., Sterling, C. R., \& Checkley, W. (2012). Effects of the 1997-1998 El Niño episode on community rates of diarrhea. American journal of public health, 102(7):e63-e69. https://doi.org/10.2105/AJPH.2011.300573

Bermúdez, A., Gómez, D., Erasso, C., \& Vélez, M. A. (2020). La cara étnica de la pandemia. NotaMacroeconómica, 24.

Dispomible en: https://www.economia.uniandes.edu.co/components/com booklibrary/ebooks/BM\%2024.pdf (Acceso marzo 2021)

Blackman, A., \& Veit, P. (2018). Amazon indigenous communities cut forest carbon emissions. EcologicalEconomics, 153(c): 56-67. https://doi.org/10.1016/j.ecolecon.2018.06.016

Blackman, A., Corral, L., Lima, E. S., \& Asner, G. P. (2017). Titling indigenous communities protects forests in the Peruvian Amazon. Proceedings of the National Academy of Sciences of the United States of America, 114(16): 4123-4128. https://doi.org/10.1073/pnas.1603290114

Brimblecombe, P. (1988). The Big Smoke: a History of Air Pollution in London Since Medieval Times, London: Methuen.

Calderón, L., \& Torres, R. (2012). Air pollution, socioeconomic status, and children's cognition in megacities: the Mexico City scenario. Frontiers in Psychology, 3:1-4. https://doi.org/10.3389/fpsyg.2012.00217

Cárdenas, E., \& Montoya, P. (1 de junio de 2020). El coronavirus y los pueblos olvidados de la región amazónica. Razón Pública. Disponible en: https://razonpublica.com/coronavirus-los-pueblos-olvidados-la-regionamazonica/ (Acceso marzo 2021)

Chay, K. Y., \& Greenstone, M. (2003).The impact of air pollution on infant mortality: Evidence from geographic variation in pollution shocks induced by a recession. Quarterly Journal of Economics, 118(3): 121-1167. Disponible en: https://www.frontiersin.org/articles/10.3389/fpsyg.2012.00217/full (Acceso marzo 2021)

Checkley, W., Epstein, L. D., Gilman, R. H., Cabrera, L., \& Black, R. E. (2003). Effects of acute diarrhea on linear growth in Peruvian children. American journal of epidemiology, 157(2): 166-175. https://doi.org/10.1093/aje/kwf179

Checkley, W., Epstein, L. D., Gilman, R. H., Figueroa, D., Cama, R. I., Patz, J. A., \& Black, R. E. (2000). Effect of El Niño and ambient temperature on hospital admissions for diarrhoeal diseases in Peruvian children. Lancet (London, England), 355(9202): 442-450. https://doi.org/10.1016/s0140-6736(00)82010-3

Checkley, W., Gilman, R. H., Black, R. E., Epstein, L. D., Cabrera, L., Sterling, C. R., \& Moulton, L. H. (2004). Effect of water and sanitation on childhood health in a poor Peruvian peri-urban community. Lancet (London, England), 363(9403): 112-118. https://doi.org/10.1016/S0140- 
Chemi, J. (2001). La globalización de la industria y la contaminación del aire urbano. EURE (Santiago), $27: 81$. http://dx.doi.org/10.4067/S0250-71612001008100002

Clark, W. de P Jr. (2003). Occupational and Environmental Diseases. Encyclopædia. New York: Microsoft Corporation; 2003. Disponible en: http://sisbib.unmsm.edu.pe/bvrevistas/anales/v65_n2/enpdf/a05.pdf (Acceso marzo 2021)

CNN. (2020). La deforestación en el Amazonas se está acelerando a pesar del coronavirus. Disponible en: https://cnnespanol.cnn.com/2020/05/15/la-deforestacion-en-el-amazonas-se-esta-acelerando-a-pesar-delcoronavirus/amp/? twitter_impression=true (Acceso marzo 2021)

Curtis, L., Rea, W., Smith-Willis, P., Fenyves, E., \& Pan, Y. (2006). Adverse health effects of outdoor air pollutants. Environment international, 32(6): 815-830. https://doi.org/10.1016/i.envint.2006.03.012

Dantas, G., Siciliano, B., França, B. B., da Silva, C. M., \& Arbilla, G. (2020). The impact of COVID-19 partial lockdown on the air quality of the city of Rio de Janeiro, Brazil. The Science of the total environment, 729, 139085. https://doi.org/10.1016/j.scitotenv.2020.13908

de Sherbinin, A., Carr, D., Cassels, S., \& Jiang, L. (2007). Population and Environment. Annual review of environment and resources, 32, 345-373. https://doi.org/10.1146/annurev.energy.32.041306.100243

Deulin R. (1997). Air Pollutants: Human Health Studies. En: Ron WN (Ed). Environmental Medicine 3a Ed. USA: Lippincott-Raven.

Du, John H. (1983). Toxicología ambiental Ed.Omega. Barcelona.

Dunn, R. R. (2010). Global mapping of ecosystem disservices: The unspoken reality that nature sometimes kills us. Biotropica, 42 (5):555-557. Disponible en: https://www.jstor.org/stable/40863785 (Acceso marzo 2021)

Ehrlich, P. R., \& Holdren, J. P. (1971). Impact of population growth. Science (New York, N.Y.), 171(3977): 12121217. https://doi.org/10.1126/science.171.3977.1212

El Tiempo. (28 de marzo de 2020). ¿Cuántas camas de UCI por persona hay en Colombia? Disponible en: https://www.eltiempo.com/datos/total-de-camas-de-cuidado-intensivo-en-colombia-478076. marzo 2021)

Ellwanger, J. H., Kulmann-Leal, B., Kaminski, V. L, Valverde-Villegas, J., Veiga, A. B., Spilki, F., \& Almeida, S. E. (2020). Beyond diversity loss and climate change: Impacts of Amazon deforestation on infectious diseases and public health. Anais da Academia Brasileira de Ciências, 92(1), 33. https://doi.org/10.1590/0001$\underline{3765202020191375}$

Elsom, D. (1990). La contaminación atmosférica Ed. Cátedra. Madrid. Disponible en: https://ddd.uab.cat/record/17227 (Acceso marzo 2021)

Foskett, E. (1999). "Historical development of environmental health in the UK” In: Bassett, W. H. Clay's Handbook of Environmental Health. London: E\&FN SPON.

Frumkin, H. (2005). Environmental Health: from global to local. USA: Jossey-Bass.

Garibay, M.G. y Curiel, A. (2005). Salud ambiental, campo de la complejidad ambiental. Revista Ideas Ambientales 2: 229-235. Disponible en:

https://www.academia.edu/1313416/SALUD_AMBIENTAL_CAMPO_DE_LA_COMPLEJIDAD_AMBIEN TAL (Acceso marzo 2021)

Gorelick, Steven (1999). "Tipping the scale: Systemic support for the large and global", The Ecologist, 29, 3:162-165.

Gouldson, A. and J. Murphy (1997). "Ecological modernization: restructuring industrial economies", MichaelJacobs (Ed.). Greening the Millenium. The New Politics of the Environment, Oxford: Blackwell.

Guerrant RL, DeBoer MD, Moore SR, Scharf RJ, Lima AA. The impoverished gut--a triple burden of diarrhoea, stunting and chronic disease. Nat Rev Gastroenterol Hepatol. 2013 Apr;10(4):220-9. https://doi.org/10.1038/nrgastro.2012.239

Herrera, D., Pfaff, A., \& Robalino, J. (2019). Impacts of protected areas vary with the level of government: Comparing avoided deforestation across agencies in the Brazilian Amazon. Proceedings of the National Academy of Sciences, 116(30):14916-14925. https://doi.org/10.1073/pnas.1802877116

Ideam (Instituto de Hidrología, Meteorología y Estudios Ambientales). (2020). Resultados monitoreodeforestación2019. 
https://www.ideam.gov.co/documents/10182/105413996/presentacionbalancedeforestacion2019/7c9323fcd0a1-4c95-b1a1 1892b162c067. (Acceso marzo 2021)

Inegi. (2019). Encuesta Nacional sobre Consumo de Energéticos en Viviendas Particulares (Encevi) 2018. Disponible en: https://www.inegi.org.mx/programas/encevi/2018/. (Acceso marzo 2021)

Instituto Nacional de Estadistica e Informatica (INEI). (2007). Censos Nacionales: XI de Poblacion y VI de Vivienda. Perfil Sociodemografico del Peru. 2da ed.Lima: INEI; 2008. Disponible en: https://www.inei.gob.pe/media/MenuRecursivo/publicaciones_digitales/Est/Lib1136/libro.pdf (Acceso marzo 2021)

Jeuland, M., Pattanayak, S. K., \& Bluffstone, R. (2015). The economics of household air pollution. Annual Review of Resource Economics, 7(1): 81-108. https://doi.org/10.1146/annurev-resource-100814-125048

Jeyaratnam J. (2000). Transfer of hazardous industries. En: Occupational Cancer in Development Countries. Lyon: IARC; 2000.

Kogevinas M, Janer G. Dioxinas y Salud. Med Clin (Barc) 2000 Dec 2; 115:740-8. Disponoble en: https://www.elsevier.es/index.php?p=revista\&pRevista=pdf-simple\&pii=S0025775300716794\&r=28 (Acceso marzo 2021)

La Dou J, Jackson RT, Howard JJ. Salud ambiental. (1999). En: La Dou J. Medicina Laboral y Ambiental. $2^{\text {a Ed. }}$ Lanata, C. F., Fischer-Walker, C. L., Olascoaga, A. C., Torres, C. X., Aryee, M. J., Black, R. E., \& Child Health Epidemiology Reference Group of the World Health Organization and UNICEF (2013). Global causes of diarrheal disease mortality in children $<5$ years of age: a systematic review. PloS one, 8(9):e72788. https://doi.org/10.1371/journal.pone.0072788

López-Feldman, A., Chávez, C., Vélez, M. A., Bejarano, H., Chimeli, A., Feres, J., \& Robalino, J. (2020). Environmental impacts and policy responses to COVID-19: A view from Latin America. Environmentaland Resource Economics, 76:471-476. https://doi.org/10.1007/s10640-020-00460-X

López-Feldman, A., Heres, D., \& Marquez-Padilla, F. (2021). Air pollution exposure and COVID-19: A look at mortality in Mexico City using individual-level data. The Science of the total environment, 756: 143929. https://doi.org/10.1016/j.scitotenv.2020.143929

López-Feldman, A., Mora, J., \& Taylor, J. E. (2007). Does natural resource extraction mitigate poverty and inequality? Evidence from rural Mexico and a Lacandona Rainforest Community. Environment and Development
Economics,
12(2):
251-269.
Disponible

https://escholarship.org/content/qt5xq0f15f/qt5xq0f15f.pdf?t=krne00 (Acceso marzo 2021)

Masera, O., Riojas, H., Pérez, R., Serrano, M., Schilman, A., Ruiz, V., \&. Berrueta, V. (2020). Vulnerabilidad a COVID-19 en poblaciones rurales y periurbanas por el uso doméstico de leña. Gobierno de México. Dispomible en: https://www.insp.mx/resources/images/stories/repositorio-inspcovid19/pdfs/Vulnerabilidad_a_COVID_por_humo_de_lena.pdf (Acceso marzo 2021)

McNeill, J. (2006). Population and the Natural Environment: Trends and Challenges. Population and Development Review 32: 183-201. Disponible en: https://onlinelibrary.wiley.com/doi/abs/10.1111/j.17284457.2006.tb00007.x (Acceso marzo 2021)

Ministerio de Salud Pública. (2020). Infografía N. 182, 27. Gobierno de Ecuador. Disponible en: https://www.gestionderiesgos.gob.ec/informes-de-situacion-covid-19-desde-el-13-de-marzo-del-2020/ (Acceso marzo 2021)

Myers, S. S., Gaffikin, L., Golden, C. D., Ostfeld, R. S., Redford, K. H., Ricketts, T. H., Turner, W. R., \& Osofsky, S. A. (2013). Human health impacts of ecosystem alteration. Proceedings of the National Academy of Sciences of the United States of America, 110(47): 18753-18760. https://doi.org/10.1073/pnas.1218656110

Nebel BJ. (1999). Ciencias Ambientales. Ecología y Desarrollo sostenible. 6a Ed. México: Prentice Hall; 1999.

Neidell M. J. (2004). Air pollution, health, and socio-economic status: the effect of outdoor air quality on childhood asthma. Journal of health economics, 23(6):1209-1236. https://doi.org/10.1016/j.jhealeco.2004.05.002

OECD (1998), Economic Globalisation and the Environment, France: Organization for Economic Co-Operation andDevelopment. Disponible en: https://www.oecd.org/sd-roundtable/papersandpublications/39365131.pdf (Acceso marzo 2021) 
Olea N, Fernández F, Araque, P Olea-Serrano. Perspectivas en disrupción endocrina. Gac Sanit 2002; 16:250-6. Disponible en: https://scielo.isciii.es/pdf/gs/v16n3/v16n3a07.pdf (Acceso marzo 2021)

Ministerio del Ambiente (MINAN). (2013). Agenda de investigación ambiental. Lima: MINAM. Disponible en: https://www.minam.gob.pe/investigacion/wp-content/uploads/sites/19/2013/10/Agenda-deInvestigaci\%C3\%B3n-Ambiental_Interiores.pdf (Acceso marzo 2021)

Porta M, Kogevinas M, Zumeta E, Sunyer J, Ribas-Fito N, Ruiz L, Jariod M, Vioque J, Alguacil J, Martin P, Malats N, Ayude D. (2002). Grupo de Trabajo sobre Compuestos Toxicos Persistentes y Salud del IMIM. Concentraciones de compuestos tóxicos persistentes en la población española: el rompecabezassin piezas y la protección de la salud pública. Gac Sanit; 16(3):257-66. Disponible en: https://scielo.isciii.es/scielo.php?script=sci_arttext\&pid=S0213-91112002000300011 (Acceso marzo 2021)

Richard, S. A., Black, R. E., Gilman, R. H., Guerrant, R. L., Kang, G., Lanata, C. F., Mølbak, K., Rasmussen, Z. A., Sack, R. B., Valentiner-Branth, P., Checkley, W., \& Childhood Malnutrition and Infection Network (2013). Diarrhea in early childhood: short-term association with weight and long-term association with length. American journal of epidemiology, 178(7): 1129-1138. https://doi.org/10.1093/aje/kwt094

Roa, S. (2020). Medidas para enfrentar al COVID-19 mejoran calidad del aire en dos ciudades ecuatorianas. MongabayLatam. Disponible en: https://www.es.mongabay.com/2020/04/menor-contaminaciondel-aire-por-coronavirus-en-quito-y-cuenca-ecuador/. (Acceso marzo 2021)

Samel JM. (1997). Community Air Pollution. En: Ron WN (Ed). Environmental Medicine 3a Ed. USA: LippincottRaven. Disponible en: https://www.redalyc.org/pdf/379/37965205.pdf (Acceso marzo 2021)

Schraufnagel, D. E., Balmes, J. R., Cowl, C. T., De Matteis, S., Jung, S. H., Mortimer, K., Perez-Padilla, R., Rice, M. B., Riojas-Rodriguez, H., Sood, A., Thurston, G. D., To, T., Vanker, A., \& Wuebbles, D. J. (2019). Air Pollution and Noncommunicable Diseases: A Review by the Forum of International Respiratory Societies' Environmental Committee, Part 2: Air Pollution and Organ Systems. Chest, 155(2): 417-426. https://doi.org/10.1016/j.chest.2018.10.041

Schraufnagel, D. E., Balmes, J. R., Cowl, C. T., De Matteis, S., Jung, S. H., Mortimer, K., Perez-Padilla, R., Rice, M. B., Riojas-Rodriguez, H., Sood, A., Thurston, G. D., To, T., Vanker, A., \& Wuebbles, D. J. (2019). Air Pollution and Noncommunicable Diseases: A Review by the Forum of International Respiratory Societies' Environmental Committee, Part 2: Air Pollution and Organ Systems. Chest, 155(2): $417-426$. https://doi.org/10.1016/j.chest.2018.10.041

Setti, L., Passarini, F., De Gennaro, G., Barbieri, P., Perrone, M. G., Borelli, M., Palmisani, J., Di Gilio, A., Torboli, V., Fontana, F., Clemente, L., Pallavicini, A., Ruscio, M., Piscitelli, P., \& Miani, A. (2020). SARS-Cov-2RNA found on particulate matter of Bergamo in Northern Italy: First evidence. Environmental research, 188:109754. https://doi.org/10.1016/j.envres.2020.109754

Shen, W., Srivastava, S., Yang, L., Jain, K., \& Schröder, P. (2020). Understanding the impacts of outdoor air pollution on social inequality: Advancing a just transition framework. Local Environment, 25(1): 1-17.

Steffen, W., K. Richardson, J. \& Rockstrom, A.. (2015). Planetary boundaries: Guiding human development on a changing planet. Science 347(6223). https://doi.org/10.1126/ science.1259855

Sukhsohale, N. D., Narlawar, U. W., \& Phatak, M. S. (2013). Indoor air pollution from biomass combustion and its adverse health effects in central India: an exposure-response study. Indian journal of community medicine : official publication of Indian Association of Preventive \& Social Medicine, 38(3): 162-167. https://doi.org/10.4103/0970-0218.116353

Vélez, M. A., Cárdenas, J., Lucumí, D., Caicedo, A., Rubiano, M. J., Arroyo, A., \& Cándelo, C. (2020a). Recomendaciones para las autoridades municipales y autoridades locales de los consejos comunitarios de comunidades para cuando termine la cuarentena nacional. Nota Macroeconómica, 19. Disponible en<. https://repositorio.uniandes.edu.co/handle/1992/41159 (Acceso marzo 2021)

Vélez, M. A., Robalino, J., Cárdenas, J. C., Paz, A., \& Pacay, E. (2020b). Is collective titling enough to protect forests? Evidence from Afro-descendant communities in the Colombian Pacific region. World Development, 128. https://doi.org/10.1016/j.worlddev.2019.104837

Weitzenfeld DH. (1992). Contaminación Atmosférica y Salud enAmérica Latina. Bol Oficina Sanit Panam; $112: 97-$ 109.Disponible en: https://iris.paho.org/handle/10665.2/16541?locale-attribute=pt (Acceso marzo 2021) 
WHO and UNEP, World Health Organization and United Nations Environment Programme (1987). Global Pollutionand Health, London: Yale University Press

Wu, X., Nethery, R. C., Sabath, B. M., Braun, D., \& Dominici, F. (2020). Exposure to air pollution and COVID-19 mortality in the United States. medRxiv. https://doi.org/10.1101/2020.04.05.20054502

Yassi A, Kjellstrom T, deKok T, Guidotti T. Salud ambiental Básica. Versión al idioma español. La Habana: Ecimed; 2008. Disponioble en: http://www.pnuma.org/educamb/documentos/salud ambiental basica.pdf (Acceso marzo 2021)

Yucra, S., Tapia, V., Steenland, K., Naeher, L. P., \& Gonzales, G. F. (2011). Association between biofuel exposure and adverse birth outcomes at high altitudes in Peru: a matched case-control study. International journal of occupational and environmental health, 17(4): 307-313. https://doi.org/10.1179/107735211799041869

Zhu, Y., Xie, J., Huang, F., \& Cao, L. (2020). Association between short-term exposure to air pollution and COVID-19 infection: Evidence from China. The Science of the total environment, 727: 138704. https://doi.org/10.1016/j.scitotenv.2020.138704 